\title{
EL DESARROLLO DE LA COMPETENCIA SOBRE VARIACIÓN TERMINOLÓGICA EN ESTUDIANTES DE TRADUCCIÓN E INTERPRETACIÓN: UNA EXPERIENCIA DE COLABORACIÓN INTERUNIVERSITARIA
}

\author{
THE DEVELOPMENT OF TERM VARIATION COMPETENCE \\ IN TRANSLATION AND INTERPRETING STUDENTS: AN \\ EXPERIENCE OF COLLABORATION BETWEEN UNIVERSITIES
}

\author{
SABELA FERNÁNDEZ SILVA \\ Pontificia Universidad Católica de Valparaíso \\ sabela.fernandez@pucv.cl
}

\section{MARIBEL TERCEDOR SÁNCHEZ \\ Universidad de Granada \\ itercedo@ugr.es}

\section{RESUMEN}

La variación terminológica constituye un problema de traducción frecuente que se da a nivel intralingüístico, por la existencia de distintos términos para referirse al mismo concepto, como a nivel interlingüístico, por la existencia de distintos equivalentes de traducción para un mismo término. En este artículo se presenta una propuesta para desarrollar la competencia sobre variación terminológica en estudiantes de traducción e interpretación en el marco de un proyecto de colaboración entre universidades. El encargo consistió en documentar la base de datos VariMed, que contiene variantes denominativas de enfermedades en español e inglés, con las variantes empleadas en el español de Chile. Se planteó un escenario de enseñanza-aprendizaje colaborativo y situado, donde se simuló un entorno profesional basado en un encargo real. A través del trabajo los estudiantes aprendieron la metodología del trabajo terminológico puntual; y desarrollaron conocimiento teórico y práctico sobre la variación terminológica y su gestión en la traducción. Como resultado del trabajo, los estudiantes elaboraron 1.170 fichas, 360 con variantes no registradas en VariMed. La propuesta didáctica se evaluó mediante una encuesta y un informe autoevaluativo. Los estudiantes encontraron mayores dificultades en la compilación de un corpus médico del español chileno, la identificación de variantes terminológicas y la validación con expertos. Por otro lado, los estudiantes reconocieron la utilidad del trabajo para su formación como traductores/intérpretes y valoraron muy positivamente su carácter situado, especialmente la colaboración científica entre universidades. 
Palabras clave: Variación terminológica; formación de traductores; competencia terminológica; trabajo terminológico puntual.

\begin{abstract}
Term variation is frequently a translation problem that manifests itself at the intralanguage level -given the existence of different term variants for the same concept and at the inter-language level- as a result of different translation equivalents for a term. This article presents a proposal for the development of term variation competence in student translators and interpreters in the framework of a collaboration project between universities. The assignment consisted on documenting the VariMed term base, containing synonyms of diseases in Spanish and English, with term variants used in Chilean Spanish. A situated and collaborative teaching-learning environment was proposed, where students had to carry out a real-life assignment in a simulated professional environment. Throughout the project, students learned the methodology of ad hoc terminology management, and developed practical and theoretical knowledge about term variation and its management in translation. As a result, students created 1170 term records, 360 corresponding to variants not yet registered in VariMed. The teaching proposal was evaluated by means of a questionnaire and a self-evaluation report. The most problems were reported in the compilation of a Chilean Spanish medical corpus, the identification of term variants and the expert validation phase. Furthermore, students found the learning experience useful for their training as translators/interpreters and evaluated positively its situated nature, especially the collaboration between universities.
\end{abstract}

Keywords: Term variation; translator training; terminological competence; ad hoc terminology work.

Recibido: 02/04/2019. Aceptado: 25/11/2019.

\title{
1. INTRODUCCIÓN
}

- $\mathrm{n}$ la traducción especializada, la gestión terminológica es una tarea crucial,

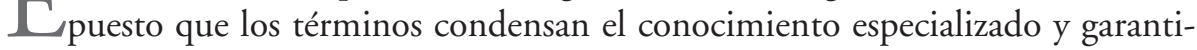
zan la concisión y precisión del discurso (Gouadec, 2007; Cabré, 1999), de manera que resulta indispensable desarrollar en los estudiantes los conocimientos y habilidades necesarios para hacer frente a los problemas terminológicos de una traducción de manera autónoma y eficaz. La competencia terminológica del traductor está a caballo entre la competencia temática, interlingüística e instrumental y atraviesa las distintas fases del proceso traductor (Montero y Faber, 2009). En la fase de comprensión, conlleva la identificación y adquisición de conocimiento especializado en el discurso, lo cual implica reconocer las unidades terminológicas 
e identificar los problemas terminológicos que se pueden presentar en la traducción. En la fase de transferencia se deben evaluar y consultar recursos de información a fin de localizar las correspondencias interlingüísticas de los conceptos. En la fase de producción, el traductor debe emplear los términos que representen el concepto de manera precisa y que se adecúen a las características de la situación comunicativa de llegada, de acuerdo con una determinada variedad geolectal, nivel de especialización o registro. De manera paralela, el traductor debe poseer conocimientos y habilidades acerca de la metodología del trabajo terminológico para crear sus propios recursos de información en forma de glosarios o base de datos, con el fin de almacenar la información terminológica obtenida en sus traducciones para reutilizarla en trabajos futuros.

En este sentido, uno de los problemas terminológicos más comunes a los que se enfrentan los traductores especializados es la variación terminológica. La variación, entendida como formas alternativas de denominar un mismo concepto especializado, es un fenómeno común y frecuente en todo tipo de textos, que obedece a factores dialectales, comunicativos o cognitivos (Freixa, 2006; Fernández, Freixa y Cabré, 2011; Tercedor, 2011). El traductor se enfrenta a un doble desafío: debe gestionar la variación intralingüística -que se da en una misma lengua como consecuencia de la diversidad de usos y usuarios de la comunicación especializada-y la variación interlingüística, que se origina por la falta de equivalencia exacta entre las lenguas y que da como resultado diversas posibilidades de traducción para una misma unidad terminológica (Kerremans, 2010). En el proceso de traducción, las diferencias interlingüísticas en un mismo género textual tienen con frecuencia una base cultural y la creatividad en la traducción se manifiesta cuando los traductores llevan a cabo los cambios necesarios para compensar y dar cuenta de las diferencias interculturales en relación con el género textual (Ulrych, 2003, p. 137). Entre estos cambios se encuentra, sin lugar a duda, el manejo de los recursos sobre la variación terminológica.

En este artículo se presenta una propuesta didáctica para desarrollar la competencia sobre variación terminológica en estudiantes de traducción e interpretación inglés-español de la Pontificia Universidad Católica de Valparaíso (PUCV) en el marco de una colaboración interuniversitaria entre el proyecto COMPETERM sobre desarrollo de la competencia terminológica de la PUCV y el proyecto COMBIMED (FFFI-59899R) sobre variación terminológica y léxico combinatorio en medicina, liderado por la Universidad de Granada ${ }^{1}$. El trabajo consistió en enriquecer la base de datos de variantes denominativas VariMed con la variedad dialectal del español de Chile, proponiendo un escenario de enseñanza-aprendi-

${ }^{1}$ El proyecto Competerm está financiado por el Fondo Científico y Tecnológico del Ministerio de Educación del Gobierno de Chile (Fondecyt Regular no1171346): http://www.competerm.org. [Consulta: 21/12/2019]. 
zaje colaborativo y situado, donde se simuló un entorno profesional basado en un encargo real. Los objetivos de aprendizaje eran, en primer lugar, aprender la metodología del trabajo terminológico puntual aplicada a la detección de variantes, al igual que tomar conciencia acerca del fenómeno de la variación terminológica, su comportamiento en el discurso y la relevancia de su correcta gestión en la traducción. Asimismo, a través de esta tarea, se pretendió también desarrollar las competencias instrumentales propias de la gestión terminológica (Kelly, 2005) y profesionales, tales como el trabajo colaborativo, la identificación y la resolución de problemas (Prieto y Fuentes, 2016).

Tras esta introducción, se presenta el marco teórico, en el que se desarrollan los fundamentos respecto a la competencia terminológica en los traductores; la variación terminológica y la base de datos de VariMed; y los entornos situados y colaborativos en la enseñanza de la traducción. En la metodología se describen los participantes, las fases del trabajo y los cuestionarios de evaluación de la tarea. En el apartado de resultados se presentan, por un lado, los resultados de la tarea de detección de variantes y, por otro, los resultados de la encuesta de evaluación del trabajo. Finalmente, se concluye el artículo con las conclusiones, en las que se esbozan los alcances, las limitaciones y el trabajo futuro.

\section{MARCO TEÓRICO}

\subsection{La competencia terminológica en la formación de traductores}

En el mercado profesional de la traducción especializada en Chile, caracterizado por un elevado nivel de trabajadores independientes (Diéguez, Lazo y Tebé, 2016), es el traductor quien a menudo tiene que gestionar la terminología en su propio trabajo. La gestión terminológica no solo implica consultar recursos existentes, sino que también crear recursos terminológicos propios y gestionarlos de tal manera "que los resultados de una investigación puedan ser almacenados para ser consultados y reutilizados en el futuro" (Bowker, 2015, p. 306). Una adecuada gestión terminológica conlleva un ahorro de tiempo y de esfuerzos que aporta un valor añadido en el demandante mercado actual de la traducción, con exigencias de calidad y tiempo muy elevadas.

La competencia terminológica de los traductores se define como "el conjunto de conocimientos, hábitos y habilidades para el trabajo con los términos que abarca las esferas lingüísticas, cognitiva y pragmático-funcional de los mismos" (Umaña y Suárez, 2011, p. 27) y se aplica tanto a la resolución de problemas terminológicos en una traducción como a la creación de recursos terminológicos propios. La dimensión cognitiva de la competencia terminológica se centra en el 
conocimiento del ámbito especializado en que se traduce, ya que "sin conocer una materia no parece ser posible identificar la terminología, y menos aún estructurarla” (Cabré, 1999, p. 195). La dimensión lingüística abarca conocimiento acerca de los sistemas lingüísticos de origen y de llegada, lo cual resulta indispensable para identificar los términos, localizar las correspondencias interlingüísticas y evaluar su corrección de acuerdo con las normas gramaticales de la lengua de llegada. La competencia pragmático-funcional implica conocer las funciones y el comportamiento de las unidades terminológicas en la comunicación especializada para que la solución terminológica sea adecuada al texto en el que se inserta y los destinatarios a quienes se dirige. A este aspecto se añade una competencia metodológica, la cual conlleva dominar las fases del trabajo terminológico puntual o sistemático, necesarias para realizar un proceso estructurado y presentar los datos de manera ordenada y eficiente; y una subcompetencia instrumental, que implica manejar los recursos de documentación y las herramientas tecnológicas para la creación de recursos terminológicos propios.

Como señalan Sánchez, Aguilar, Mesa y Solé (2009, p. 110), el desarrollo de la competencia terminológica en traductores e intérpretes debería focalizarse en los siguientes objetivos de aprendizaje: a) adquirir el conocimiento teórico necesario para comprender y desarrollar el trabajo terminológico; b) adquirir las habilidades metodológicas para realizar un trabajo terminológico sistemático; c) adquirir las habilidades metodológicas para resolver problemas terminológicos específicos.

En efecto, los niveles de implicación terminológica de un traductor descritos por Cabré (1999), que se relacionan con el nivel de competencia terminológica alcanzado a través de la formación, incluyen como criterio distintivo el conocimiento de la metodología de trabajo terminológico sistemático aplicada a la creación de recursos terminológicos propios.

\subsection{La variación terminológica como problema de traducción}

La variación terminológica es un fenómeno común en los lenguajes de especialidad, que surge por las distintas formas de denominar los conceptos especializados, donde las variantes mantienen una relación semántica y conceptual con el término original (Daille, Habert, Jacquemin y Royauté 1996, p. 201). Asimismo, obedece a factores dialectales, cognitivos, comunicativos o interlingüísticos. Por un lado, los factores dialectales se deben a diferentes orígenes geográficos o características socioprofesionales de los hablantes. Por otro lado, la denominación a través de distintas formas lingüísticas revela matices cognitivos y expresivos que justifican una selección concreta por parte del emisor. De esta forma, las variantes pueden reflejar diferentes maneras de comprender el concepto por parte de los emisores 
(Tercedor, 2011) y, del mismo modo, la variación en un mismo texto se emplea como recurso cognitivo de progresión del conocimiento o como recurso expresivo para evitar la repetición (Fernández, 2018). Entre las causas comunicativas se encuentran el nivel de especialización de los interlocutores y del texto, ambos aspectos fundamentalmente cambiantes como consecuencia de los procesos de traducción. De esta manera, un encargo de traducción puede implicar un cambio de registro, que conlleva la selección de una variante terminológica diferente a la correspondiente al nivel de especialización del texto original (TO). Las causas interlingüísticas también son importantes, puesto que se originan en el contacto entre lenguas por motivos sociopolíticos o geográficos y determinan, por ejemplo, la mayor o menor adopción y aceptación de préstamos o calcos en una comunidad concreta. La traducción exige un continuo proceso de recontextualización, como en el caso de lectores legos, para dar respuesta al conocimiento de los lectores (Calsamiglia y Van Dijk, 2004, p. 371). Los procesos de traducción e interpretación conllevan aspectos cognitivos y textuales que determinan la selección léxica a nivel intratextual e intertextual. La existencia de variación terminológica representa un doble desafío para el traductor, ya que debe gestionar la variación intralingüísti$\mathrm{ca}$-que se da en una misma lengua o texto especializado como consecuencia de la diversidad de usos y usuarios de la comunicación especializada- y la variación interlingüística-que se origina por la falta de equivalencia exacta entre las lenguas y que da como resultado diversas posibilidades de traducción para una misma unidad terminológica- (Kerremans, 2010).

Por lo tanto, el traductor debe desarrollar una serie de competencias para gestionar el fenómeno de la variación terminológica en las distintas fases del proceso traductor. En la fase de comprensión debe ser capaz de identificar las variantes terminológicas que refieren a los mismos conceptos en el interior de un texto para adquirir el conocimiento representado en el mismo. En la fase de producción debe ser capaz de emplear la variante que corresponde con los parámetros dialectales (diatópicos, diacrónicos y diastráticos) y funcionales (nivel de lengua, de especialización y registro) del texto de llegada. Asimismo, conocer las motivaciones cognitivas y comunicativas de la variación y su funcionalidad en el discurso especializado resulta significativo para decidir, por ejemplo, si se mantiene la variación presente en el texto fuente, se modifica o se elimina (Suárez, 2004). En definitiva, muchos de los problemas de traducción derivados de la variación terminológica exigen creatividad por parte del traductor, un elemento fundamental en el proceso traductor, cuya selección acertada determinará el éxito del texto. De este modo, por ejemplo, se selecciona un hiperónimo para aligerar la carga cognitiva en el 
lector, una vez se haya introducido el término específico que se necesita, también se escogen siglas o la forma desarrollada del término o una paráfrasis en función del grado de familiaridad que se presupone en el destinatario del texto, o se recurre a las formas eponímicas cuando estas son lo suficientemente extendidas y no provocan confusión.

El conocimiento respecto a la variación terminológica se vuelve crucial ya que los recursos terminológicos disponibles para el traductor muchas veces no incluyen variantes o, cuando las documentan, no proporcionan la información suficiente para orientar su empleo por parte de los traductores (Collet, 2004; Fernández y Becerra, 2015). Por ello, resulta necesario también dotar a los traductores o intérpretes de las herramientas metodológicas para registrar y representar adecuadamente la variación terminológica en los recursos creados por ellos mismos.

\subsubsection{La base de datos VariMed y su utilidad para traductores y otros profesiona- les de la comunicación}

VariMed es un recurso fruto de dos proyectos de investigación ${ }^{2}$ que contiene una base de datos terminológica acerca de enfermedades, signos y síntomas, con 1.299 conceptos, más de 5.800 variantes en inglés y español y cerca de 2.000 imágenes que ilustran los conceptos. El recurso fue diseñado específicamente para recopilar variantes terminológicas empleadas en medicina en las distintas variedades del español y el inglés, así como en textos de diferentes registros comunicativos y niveles de especialización. La interfaz de consulta (ver Figura 1) permite realizar búsquedas personalizadas por información conceptual, partes del cuerpo, imágenes, registro, etiquetas de uso y dimensiones conceptuales, tanto en inglés como en español.

El recurso está dirigido a traductores y redactores médicos que quieran escribir textos para distintos tipos de usuarios, así como divulgar conocimiento (Tercedor, 2017). Además, VariMed permite la investigación lingüística al proporcionar listas de términos clasificados según distintos criterios. Mediante la opción de búsqueda por conceptos se obtiene una imagen del concepto, sus diferentes denominaciones, información definicional y sobre usos y dimensiones de conceptualización.

${ }^{2}$ FFI-2011-23120 y FFI-2014-51899R. "Léxico Combinatorio en Medicina: Cognición, Texto y Contexto" (CombiMed) (FFI2014-51899-R) e "Investigación de la variación denominativa como fenómeno cognitivo y comunicativo" (VariMed) (FFI2011-23120), proyectos concedidos por el Ministerio de Economía y Competitividad del Gobierno de España. 


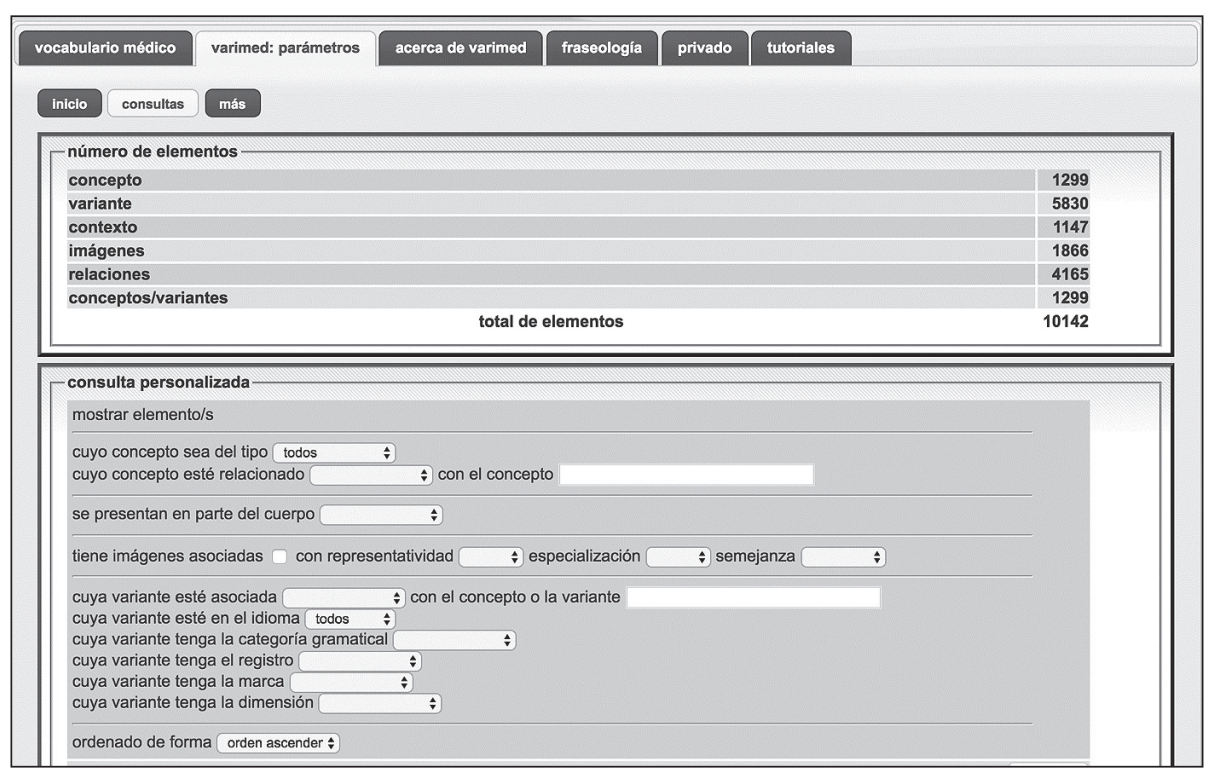

Figura 1. Interfaz de consulta personalizada de VariMed.

Para cada variante terminológica se incluye información sobre la categoría gramatical (sustantivo, adjetivo, verbo, unidad fraseológica nominal), el registro (formal, informal, expertos-informal, neutro y niños), el uso geográfico y las etiquetas de uso. Las etiquetas de uso ofrecen información acerca de una variante en concreto, ya sea relativa a su origen (préstamo, calco, origen grecolatino), a su procedimiento de formación (abreviatura, acortamiento, epónimo) u otras (término cultural, neologismo, variante más empleada). Dado el enfoque descriptivo de VariMed, también se incluyen variantes que pueden denotar un uso incorrecto, tales como falsos amigos, incorrecciones ortográficas, usos no recomendados, términos obsoletos, etcétera. Este tipo de información de uso puede ser de gran valor para tareas de redacción y traducción. 


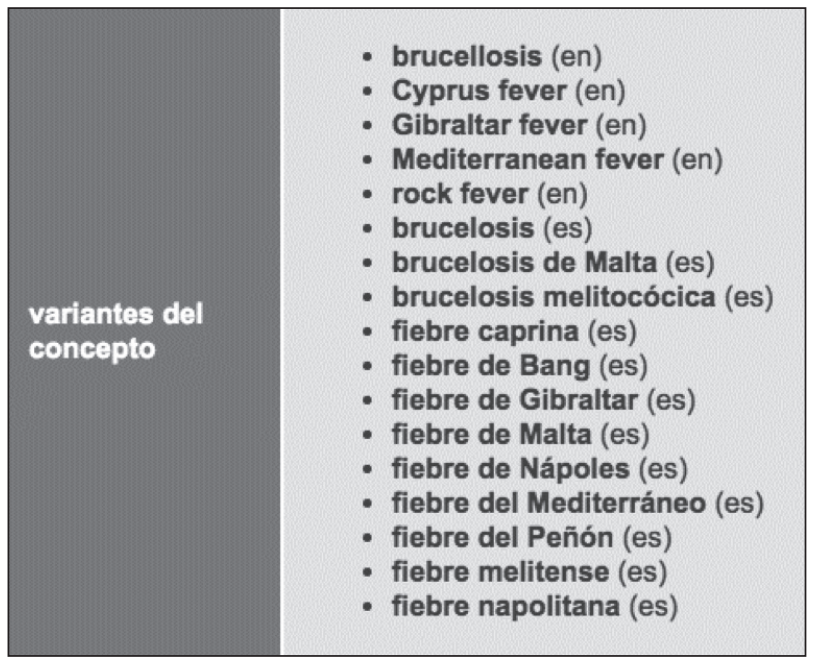

Figura 2. Variantes del concepto BRUCELOSIS recogidas en VariMed.

Es interesante recalcar que en muchas de las variantes convergen distintos factores de variación. Por ejemplo, los términos culturales corresponden, a menudo, a términos que denotan un uso geolectal. Por ejemplo, las diferentes denominaciones del concepto BRUCELOSIS, recogidas en la Figura 2, denotan un uso asociado con motivaciones sociogeográficas, muchas de ellas con una motivación cognitiva, además de cultural. Asimismo, se ha incorporado un módulo de fraseología que recoge una recopilación de refranes, frases hechas y locuciones verbales relacionadas con la medicina.

\subsection{La importancia del trabajo colaborativo y situado en la formación en tra- ducción}

Es bien sabido que la traducción profesional está siendo objeto de cambios profundos que influyen en la forma de trabajar de los traductores, que se ha vuelto cada vez más colaborativa; en las herramientas, que ofrecen mayor eficiencia y disponibilidad de acceso; e incluso en los textos, que son pretraducidos por sistemas de traducción automática en muchas ocasiones, lo que significa nuevos puntos de atención en el trabajo del traductor y en la mayor concreción de elementos problemáticos. Las herramientas de apoyo al traductor deben ofrecer soluciones tanto a las necesidades surgidas de la decodificación del texto de origen, como a las que se derivan de la producción textual asociada al texto meta (Tercedor, Faber y López, 2012, p. 181). 
Por una parte, Prieto y Fuentes (2016) apuntan a la tecnología como motor de cambio de los nuevos escenarios colaborativos; por otra parte, Hurtado (2007, p. 174) señala que la utilidad de los nuevos enfoques de enseñanza-aprendizaje se encuentra en el desarrollo de habilidades respecto a los mercados de trabajo, el uso de fuentes documentales y el manejo de herramientas para la práctica profesional. Los enfoques situados del aprendizaje conciben que el aprendizaje forma parte de un contexto, una actividad y una cultura (Brown, Collins y Duguid, 1989). En palabras de Risku (2016), "we do not just learn because we absorb 'facts' as 'information', but because we navigate with others in a given environment and so learn to act in specific situations" (p. 16). Estos enfoques situados (Vienne, 1994; Risku, 2002; Gouadec, 2007) y colaborativos (Kiraly, 2000) en los procesos de enseñanza-aprendizaje de la traducción incluyen la simulación de entornos profesionales basados en proyectos y encargos reales, que permiten un acercamiento a situaciones auténticas de ambientes profesionales. El éxito de las metodologías de enseñanza se obtiene si facilita la colaboración e interacción y si deriva en la capacidad de aplicarlo a distintos contextos reales (Hendricks, 2001; Ünal y Zeynep, 2010) y diferentes culturas.

Autores como Robinson, López y Tercedor (2008), Kiraly (2000) y Risku (2002) consideran que los entornos colaborativos propician el aprendizaje situado al favorecer la interacción e interdependencia entre los miembros de los equipos. Los equipos se configuran para llevar a cabo tareas concretas o proyectos amplios, en función de las competencias y el alcance del trabajo. Desde la perspectiva socioconstructivista, el enfoque situado corresponde a la forma habitual de trabajo en el aula de traducción y tiene, según Olvera et al. (2007, p. 521), los siguientes objetivos:

a. familiarizar a los estudiantes con los métodos de trabajo habituales en agencias de traducción;

b. desarrollar el teletrabajo, el autoaprendizaje y la interdisciplinariedad en los equipos;

c. proporcionar un entorno de trabajo con las herramientas necesarias para profesores e investigadores, de modo que se incorporen las tecnologías de la información y comunicación en el aula y se reproduzca así el contexto de trabajo profesional.

Estos enfoques tienen su origen en la zona de desarrollo próximo de Vygotsky (1978), quien reconoce la riqueza de las múltiples habilidades y los múltiples enfoques. Como cabe esperar, un entorno en el que se negocian significados, matices, focos, etcétera, es óptimo para una actitud proactiva que favorece el desarrollo de competencias terminológicas, que facilitan un mayor conocimiento de la variación terminológica en relación con el dominio de los factores que intervienen en la selección léxica en entornos interculturales e interlingüísticos. 


\section{LA INVESTIGACIÓN}

\subsection{El proyecto de colaboración}

Se propuso un trabajo que consistía en documentar las variantes denominativas en el español de Chile de la base de datos terminológicos VariMed. Este trabajo se desarrolló en el marco del proyecto final de la asignatura Terminología 3 de la carrera de traducción e interpretación inglés-español de la Pontificia Universidad Católica de Valparaíso. Los participantes fueron 49 estudiantes de traducción e interpretación en el $8^{\circ}$ semestre de la carrera, divididos en dos grupos. Se establecieron los siguientes objetivos de aprendizaje:

1. Aprender la metodología de trabajo terminológico puntual.

2. Tomar conciencia acerca de la variación terminológica -en especial la variación geolectal- y su relevancia para la traducción o interpretación.

3. Desarrollar estrategias de documentación en una variedad lingüística poco representada en los recursos terminológicos, como lo es el español de Chile.

4. Realizar un trabajo colaborativo, mediante la organización en equipos de trabajo con distintos roles en su interior.

Se planteó como un trabajo colaborativo y situado debido a que, por un lado, los estudiantes tuvieron que llevar a cabo un encargo terminológico auténtico, destinado a enriquecer una base de datos terminológicos existente en el marco de una colaboración científica internacional; y, por otro, trabajaron en un ambiente colaborativo facilitado por el uso de herramientas tecnológicas, donde el "aprendizaje se construye a través de la interacción, la responsabilidad y la dependencia positiva entre miembros del equipo" (Prieto y Fuentes, 2016, p. 6).

\subsection{Etapas del trabajo}

El proyecto se secuenció según las etapas metodológicas del trabajo terminológico multilingüe propuestas por Cabré y Tebé (2005), adaptadas a la búsqueda de equivalencias intralingüísticas (variantes denominativas en el español chileno), a las características particulares de la base de datos de VariMed y al contexto de enseñanza-aprendizaje. El trabajo se desarrolló en un periodo de 6 semanas, que comprendió 12 sesiones de clase presenciales (18 horas) y trabajo adicional fuera del aula (18 horas aproximadamente). Los estudiantes ya poseían conocimientos teóricos básicos respecto a la variación terminológica y las competencias acerca de documentación, gestión terminológica y manejo de herramientas computacionales necesarias para desarrollar el proyecto, que habían adquirido en las asignaturas 
Terminología 1 y 2 . Antes de comenzar el trabajo, debieron documentarse con respecto a la base de datos y el proyecto VariMed y recibieron instrucciones que detallaban las distintas etapas y las tareas correspondientes a cada una de ellas.

\section{Etapa 1: Preparación del trabajo}

Organización de los equipos de trabajo: los estudiantes se dividieron en grupos de 2 a 4 integrantes y asumieron roles de coordinación al interior del grupo (encargado/a de documentación, búsqueda de variantes, validación), a pesar de que todos los integrantes tuvieron que participar en cada una de las tareas. También se estableció el sistema de comunicación y las herramientas de trabajo (trabajo en la nube con las herramientas de edición de documentos de texto y hojas de cálculo de Google, el sistema de almacenamiento de archivos de Drive y el chat de Google, además del Aula Virtual de la asignatura). Finalmente, se confeccionó el cronograma de trabajo.

Análisis de la nomenclatura de partida: se entregó a los estudiantes la nomenclatura de VariMed (1.125 conceptos y 2.529 variantes en el español de España) y se pidió que seleccionaran 5 conceptos pertenecientes a una subárea de la medicina por cada miembro del equipo. Puesto que VariMed no tiene una clasificación por subáreas temáticas, se realizaron búsquedas de conceptos asociados con partes del cuerpo, bajo la premisa de que las especialidades de la medicina se relacionan con enfermedades que afectan a determinadas partes del cuerpo (como, por ejemplo, 'ojos' a oftalmología; 'cuello del útero', 'ovarios', 'útero' y 'mama' a ginecología, etcétera).

Diseño y compilación del corpus: esta fase tiene por objetivo encontrar fuentes documentales que sean representativas de la subárea de la medicina y de la variedad lingüística seleccionadas. En primer lugar se establecieron los siguientes criterios de compilación del corpus (Buendía y Ureña, 2010; Montero, Faber y Buendía, 2011; Gómez y Vargas, 2003):

- Géneros textuales: se incluyeron géneros textuales académicos y profesionales de diferentes niveles de especialización (tesis, manuales, monografías, artículos científicos, artículos de divulgación, folletos).

- Rango temporal: se estableció un rango temporal amplio, entre 1950 y 2017.

- Contenido: se estableció la medicina general y las ramas de la medicina correspondientes a la selección conceptual de cada grupo.

- Lengua: se acotó al español de Chile. Para ello, se cauteló la ubicación de la fuente (dominio .cl o páginas alojadas en Chile), la editorial o el lugar de publicación (revistas, asociaciones u organizaciones de Chile) y la autoría (se veri- 
ficó la nacionalidad o el lugar de trabajo de las personas autoras de los textos).

- Autoría: se incluyeron textos que explicitaran la autoría individual o colectiva, a fin de garantizar la variedad lingüística del español de Chile y el grado de fiabilidad del documento, para garantizar el uso correcto de los términos.

Como complemento al corpus textual, los estudiantes también realizaron búsquedas de recursos lexicográficos de medicina (diccionarios, glosarios, bases de datos) con el fin de complementar la información proporcionada por el corpus, sobre todo en lo referente a las definiciones de los conceptos.

Para la documentación, los estudiantes llevaron a cabo búsquedas en sitios web específicos (facultades de medicina de universidades chilenas, Colegio Médico de Chile y colegios médicos regionales, repositorio de revistas científicas Scielo Chile) o en motores de búsqueda generales y especializados (Google Scholar), en los cuales se debía restringir la búsqueda a Chile mediante las herramientas de búsqueda avanzada. A continuación, cada grupo elaboró una lista de los documentos seleccionados donde figuraba el nombre completo, el link, el tipo de subcorpus -normativo, normalizado, lexicográfico/terminológico o textual según la clasificación de Cabré y Tebé (2005) - y la fiabilidad de la fuente -baja, media o alta-. Finalmente, cada grupo subió el corpus en .pdf y .txt a una carpeta compartida para conformar un corpus unificado de mayor tamaño.

Revisión de la ficha terminográfica de VariMed: se realizó una revisión de la ficha terminográfica de VariMed a fin de identificar qué información se debía consignar acerca de cada variante y valorar la inclusión o exclusión de algún campo. Se decidió el formato de ficha que figura en la Tabla I.

\section{Etapa 2: Trabajo terminológico}

Esta etapa se centró en el trabajo de búsqueda y registro de variantes, así como de identificación y resolución de problemas terminológicos. Para la búsqueda de variantes nuevas en el corpus del español de Chile, los estudiantes aplicaron las siguientes estrategias mediante el programa de explotación de corpus Textstat ${ }^{3}$ :

Análisis de los contextos de aparición de las variantes de VariMed: esta estrategia se fundamenta en la idea de que en ocasiones las variantes de un mismo concepto coocurren en el discurso, o bien explícitamente por medio de marcadores, o bien como mecanismo de correferencia léxica. En el siguiente ejemplo, el análisis del contexto de afasia de Broca, variante recogida en VariMed, muestra la existencia de otras 5 variantes, indicadas expresamente con la expresión "otros nombres que ha recibido son":

\footnotetext{
${ }^{3}$ Disponible en: http://neon.niederlandistik.fu-berlin.de/en/textstat/ [Consulta: 21/12/19].
} 
- Afasia de Broca: Fue denominada inicialmente por Paul Broca como afemia, otros nombres que ha recibido son afasia motora eferente o cinética, afasia expresiva, afasia verbal o afasia sintáctica. (González y Toledo, 2007, p. 18)4.

- Búsqueda por marcadores explícitos de variación: se llevaron a cabo búsquedas a partir de marcadores de variación denominativa explícita, aquellos "marcadores discursivos que vehiculan equivalencia” (Suárez, 2004, p. 2). Se comenzó con una lista inicial de marcadores explícitos de variación ("[también] llamado', 'denominado', 'conocido como', 'o', paréntesis; Suárez, 2004), que se fue ampliando a medida que las búsquedas arrojaban otros marcadores explícitos (por ejemplo, 'otros nombres que ha recibido').

- Búsqueda por constituyentes de términos compuestos (Fernández, 2016): las variantes se dividieron en constituyentes y se examinaron las ocurrencias de cada constituyente para identificar nuevas variantes. Por ejemplo, para la variante delirio de parasitosis, la búsqueda por la base delirio arrojó las variantes delirio de ectoparasitosis, y la búsqueda por la extensión parasitosis permitió identificar la variante parasitosis psicogénica.

Durante el proceso de búsqueda de variantes, los estudiantes fueron resolviendo dudas presencialmente durante la clase y a través del foro del Aula Virtual. Se hace referencia a los problemas encontrados en el apartado de resultados. Con el fin de preparar los datos para la validación, se registraron las nuevas variantes que aparecieron en el corpus del español de Chile, así como también los casos en que las variantes ya registradas en VariMed aparecían también en el corpus, junto con los contextos y las fuentes.

\section{Etapa 3: Revisión y llenado de fichas}

En esta etapa, los datos hallados fueron validados por especialistas. Cada grupo se puso en contacto con especialistas de nacionalidad chilena del área de la salud para validar las variantes encontradas. Se definió como especialista a un médico o médica en ejercicio, egresado/a o estudiante de medicina de último(s) año(s), aunque dada la dificultad para encontrar especialistas también se aceptó, en algunas ramas de la medicina, otros profesionales del área de la salud, tales como enfermeros/as, psicólogos/as, fonoaudiólogos/as, nutricionistas, etcétera. Cuatro grupos (de un total de 20) no pudieron contactar con especialistas, de modo que la validación se hizo mediante el corpus y la consulta de obras de referencia. La validación se realizó de manera presencial o virtual, según la disponibilidad del especialista. Se pidió

${ }^{4}$ González, Rafael y Toledo, Lilian. 2007. Diagnóstico diferencial de las afasias. (Guía docente). Santiago: Universidad de Chile. Disponible en: http://repositorio.uchile.cl/bitstream/handle/2250/123326/Guia_Docente_Afasia_2007.pdf [Consulta: 20/12/19]. 
que respondiera si las variantes proporcionadas aludían al concepto en cuestión, si se utilizaban en Chile y si conocía otras variantes para dicho concepto. Se preparó una tabla con la siguiente información para cada variante: 1) el concepto al que hace referencia; 2) la variante; 3) un contexto de aparición; 4) el link a la fuente completa; 5) un espacio donde debía responder sí o no; 6) un campo para que incluyera otras variantes; 7) una sección de observaciones para que el especialista estableciera libremente la información que estimara conveniente.

Una vez validados los datos, se procedió a elaborar las fichas lexicográficas, con la siguiente información para cada variante, tal y como se muestra en el ejemplo de la Tabla I.

Tabla I. Estructura de la ficha empleada en el trabajo.

\begin{tabular}{|c|c|}
\hline Variante & enfermedad celíaca \\
\hline Concepto & CELIAQUÍA \\
\hline Categoría gramatical & Unidad fraseológica, $\mathrm{f}$. \\
\hline Idioma & ES \\
\hline Registro & Neutro \\
\hline Otras marcas & Nomenclatura ICD-10 / CIE-10 \\
\hline Uso geográfico & ES-CL \\
\hline Observaciones & Su uso también se documentó en Argentina. \\
\hline Contexto 1 & $\begin{array}{l}\text { "Un estudio de cohorte retrospectivo de } 679 \text { pacientes con } \\
\text { enfermedad celíaca confirmada por biopsia, que se centró en } \\
\text { las modificaciones del peso corporal en sujetos siguiendo una } \\
\text { DLG, mostró que el IMC promedio fue de } 24 \text {, con un bajo } \\
\text { número de sujetos con sobrepeso y obesidad." }\end{array}$ \\
\hline Fuente 1 & $\begin{array}{l}\text { Araya, M. y Bascuñán, K. (2014). Enfermedad celíaca. } \\
\text { Una mirada actual. Revista chilena de pediatría, 85(6), 658- } \\
665 \text {. Disponible en: https://dx.doi.org/10.4067/S0370- } \\
41062014000600002\end{array}$ \\
\hline Contexto 2 & $\begin{array}{l}\text { ES-AR: "La Enfermedad Celíaca es una condición permanen- } \\
\text { te de intolerancia al gluten contenido en diversos alimentos, } \\
\text { que ocurre en individuos genéticamente predispuestos (niños } \\
\text { y adultos), y se manifiesta como una enteropatía mediada por } \\
\text { mecanismos inmunológicos." }\end{array}$ \\
\hline Fuente 2 & $\begin{array}{l}\text { Ministerio de Salud Argentina (2013). Guía de práctica clínica } \\
\text { sobre diagnóstico y tratamiento de la enfermedad celíaca en el } \\
\text { primer nivel de atención. Disponible en: http://www.msal.gob. } \\
\text { ar/celiacos/pdf/2013-03-08 guia-practica-clinica-enfermedad- } \\
\text { celiaca.pdf }\end{array}$ \\
\hline
\end{tabular}




\subsection{Instrumentos de evaluación de la propuesta didáctica}

A fin de evaluar la propuesta didáctica, se aplicaron dos instrumentos:

1. Un cuestionario en línea, que los estudiantes completaron anónimamente al final del proyecto, con el objetivo de evaluar el grado de cumplimiento de los objetivos y su nivel de satisfacción con la tarea.

2. Un informe autoevaluativo, que cada grupo entregó por escrito a la profesora como parte de la evaluación del trabajo final, en el que debían exponer los problemas hallados durante la realización del trabajo, plantear las estrategias aplicadas para su resolución y emitir una valoración personal acerca de la utilidad del proyecto en relación con los objetivos de aprendizaje y su formación como traductores o intérpretes.

\section{RESULTADOS}

En este apartado se presentan, en primer lugar, los resultados del trabajo de búsqueda de variantes denominativas realizado por los estudiantes y, en segundo lugar, los resultados de evaluación de la propuesta didáctica.

\subsection{Identificación de variantes denominativas}

El trabajo de reconocimiento de variantes arrojó 1.170 fichas de variantes denominativas correctas, una vez realizada la validación de los especialistas y la corrección por parte de la profesora. Por un lado, un 68 \% (801) de las variantes identificadas por los estudiantes en el corpus del español de Chile ya estaban registradas en VariMed y, por lo tanto, son de uso común en España y Chile. Por otro lado, un $32 \%$ (369) corresponde a variantes denominativas que no se hallaban registradas en VariMed y, por consiguiente, podrían ser susceptibles de constituir variantes dialectales diferenciales del español de Chile con respecto al español peninsular.

Tras analizar la tipología de estas nuevas variantes documentadas en Chile, se encontraron casos que se corresponden con usos diferenciados geográficamente y otros en los que el término también se emplea en España, pero no fue documentado en VariMed. A continuación, se hace referencia a algunas de las categorías más comunes halladas en el corpus: 


\section{Variantes gráficas}

Se documentaron diferentes tipos de variantes en las que se da un cambio gráfico en relación con las variantes registradas en VariMed. Es el caso de las siglas, donde se encontraron tanto fenómenos de siglación discursiva (cuando se establece la equivalencia con la forma desarrollada en el mismo documento mediante un marcador explícito, generalmente paréntesis, y su uso se restringe al contexto discursivo inmediato), tal como la variante $A A$ del concepto APENDICITIS AGU$\mathrm{DA}^{5}$, como también de otras siglas lexicalizadas y de uso extendido en el discurso médico, tal como TEA, correspondiente al concepto TRASTORNO DEL ESPECTRO AUTISTA. Asimismo, se hallaron variantes ortográficas, como ponfólix (CL) frente a pomfólix (ES), neumonia (CL) frente a neumonía (ES), o beri-beri (CL) frente a beriberi (ES). En este grupo de variantes formales se consideró que la mayoría de los usos son comunes en ambos países, a excepción de ciertas variantes con acentuación distinta (por ejemplo, neumonia [CL]) o siglas en inglés cuyo uso está más extendido en Chile que en España, tal como PLMD (CL) de periodic limb movement disorder. Esta observación resulta coherente con estudios que indican la alta permeabilidad del español de Chile hacia la adopción de anglicismos (Gerding, Fuentes y Kotz, 2012) y una mayor influencia del inglés médico en países latinoamericanos (Navarro, 2001).

\section{Variantes morfosintácticas}

En este grupo se documentaron variaciones por presencia o ausencia de artículo, tal como úlcera del duodeno (CL)/ úlcera de duodeno (ES); cambios de preposición, como trastorno por ansiedad social (CL)/ trastorno de ansiedad social (ES); o cambios de morfemas sinonímicos, tal como depresión monopolar (CL)/ depresión unipolar (ES). En los casos mencionados, se ha identificado una tendencia en el español de Chile a utilizar con mayor frecuencia la preposición por y a en los sintagmas terminológicos como trastorno por ansiedad social (CL)/ trastorno de ansiedad social (ES) o sangrado por la nariz (CL)/ sangrado de la nariz (ES) y cáncer a la próstata (CL) frente a cáncer de próstata (ES) o cáncer al pulmón (CL) frente a cáncer de pulmón (ES). También se presentan algunos casos de variantes morfológicas más frecuentes en una variedad dialectal, tal como resfrío común (CL) versus resfriado común (ES). Además, se registraron alternancias entre las estructuras N-SP y NA (intoxicación por alimentos [CL]/ intoxicación alimentaria [ES]) o cambios en el orden de los constituyentes -sindrome fetal alcohólico (CL)/ sindrome alcohólico fetal (ES)-; sin embargo, en estos casos se considera que estas variantes no corresponden a un uso dialectal diferenciado.

\footnotetext{
${ }^{5}$ En este caso se utiliza las mayúsculas para representar los conceptos.
} 


\section{Reducciones}

En el corpus del español de Chile se encontraron variantes que constituyen reducciones o ampliaciones con respecto a las variantes documentadas en el español de España, tanto de la base -somatización (CL)/ trastorno de somatización (ES) - como de la extensión -trastorno del ánimo (CL)/ trastorno del estado de ánimo (ES)-. Se estima que las reducciones tampoco son casos de usos dialectales diferenciales, ya que esta variación se halla más asociada a razones discursivas, como evitar la repetición, o estrategias explicativas, como enfatizar algún aspecto del concepto en la denominación.

\section{Cambios léxicos}

Las variantes por cambios léxicos corresponden a la categoría más abundante, heterogénea y también más interesante desde el punto de vista de la variación dialectal, ya que en esta clasificación se concentran numerosos usos distintivos entre España y Chile. Por un lado, destacan las variantes en Chile que son calcos del inglés, frente a otros usos vernáculos del español de España, tal como transpiración (CL) frente a sudoración (ES), o desorden de pánico (CL)/crisis de pánico (ES) y desorden de la personalidad (CL)/ crisis de personalidad (ES), pese a que en ciertos casos la forma no calcada se documentó en Chile y no en España (por ejemplo, sindrome poliglandular autoinmunitario). Por otro lado, también se hallaron algunos usos diferenciales correspondientes a denominaciones populares de enfermedades, vinculadas a creencias acerca del origen de esta, como el caso de fuego y calor al estómago (CL) como variante de AFTA.

En síntesis, la documentación de las variantes denominativas de enfermedades, síntomas y signos en el español de Chile ha arrojado como resultado que muchos de los usos son comunes en España y Chile y que existen numerosas otras causas de variación que explican la existencia de variantes para un mismo concepto en ambos países, tal como el nivel de especialización, la variación estilística, la alternancia entre formación culta y vernácula, la interferencia de otros códigos (fundamentalmente el inglés y, en menor medida, las nomenclaturas en latín) o la convivencia de múltiples criterios de clasificación de un mismo concepto que se reflejan en la denominación. 


\subsection{Evaluación de la propuesta didáctica}

\subsubsection{Evaluación respecto a las dificultades encontradas durante la realización del trabajo: informe final}

En el apartado de discusión del informe final, los estudiantes expusieron, por un lado, los principales problemas encontrados durante la realización del trabajo y las estrategias aplicadas para su resolución, y por otro, emitieron una evaluación personal acerca de la utilidad del proyecto para su formación como traductores o intérpretes. Los principales problemas se concentraron fundamentalmente en tres etapas del trabajo: 1) recopilación del corpus representativo del español de Chile; 2) Identificación de variantes terminológicas y 3) validación con especialistas.

1) En la recopilación del corpus, los problemas se debieron a la escasa disponibilidad de textos de algunas especialidades de medicina redactados en la variedad del español de Chile. En ocasiones, aunque los textos estuvieran alojados en sitios web chilenos, no se podía determinar la autoría y, por consiguiente, la nacionalidad de los autores; en otras, se identificaba que el autor/a no era de nacionalidad chilena. Asimismo, ciertos textos que sí tenían autoría chilena correspondían a fiabilidad media o baja, ya que pertenecían a géneros digitales divulgativos (por ejemplo, blogs personales, sitios web comerciales) y no se podían incluir como fuentes fiables. Finalmente, algunos estudiantes también señalaron problemas de accesibilidad a los documentos en formato digital, porque las url se hallaban desactualizadas o los textos estaban digitalizados como imagen. Las estrategias de resolución a estos problemas consistieron en ampliar el corpus mediante sucesivas búsquedas en Internet, aplicar con mayor rigor los criterios de selección de los textos y, en última instancia, recurrir a la validación de los expertos.

2) En la fase de identificación de variantes, los problemas se centraban en discernir cuándo dos unidades léxicas eran realmente sinónimas o mantenían otro tipo de relación semántica, como la hiponimia, hiperonimia o cohiponimia. En el caso de unidades poliléxicas, este problema se solapaba con el de segmentación de las unidades en el discurso (dónde empieza y termina una unidad terminológica), al igual que de discriminación entre unidades terminológicas (léxicas de origen o lexicalizadas) y otras secuencias discursivas que representan también al concepto, pero que se encuentran por encima del nivel léxico, como la paráfrasis, las definiciones, las explicaciones, etcétera. Con el fin de resolver estos problemas, los estudiantes debieron corroborar el uso de la variante en otras fuentes documentales, lexicográficas o recurrir a la validación de los expertos. Por último, algunos estudiantes también señalaron que, en ciertos casos, se presentaban usos erróneos 
de variantes por parte de los/as autores/as de los textos, que se detectaban primordialmente en la fase de consulta a especialistas.

3) La fase de validación con expertos fue la que más dificultades entrañó a los estudiantes, tanto operativas como metodológicas. En primer lugar, numerosos estudiantes manifestaron la dificultad para encontrar especialistas del área de la salud con disponibilidad para colaborar, lo que implicó en ocasiones cambiar al experto o preparar material con el fin de realizar la entrevista de manera no presencial. A este aspecto se añadió la dificultad para explicar la tarea; por ejemplo, aclarar las diferencias entre concepto, término o variante. Otro grupo de problemas se dio por la discrepancia entre la información proporcionada por los textos y la opinión de los expertos en cuanto al uso de las variantes en Chile o su correspondencia con el concepto en cuestión. En ocasiones, las variantes aparecían documentadas en textos chilenos, pero el/la especialista no validaba su uso en Chile; en otras, el autor/a del texto había empleado una variante como sinónimo, pero el experto/a veía una diferencia conceptual. En estos casos de discrepancia, los estudiantes tuvieron que aplicar su propio criterio, lo que en ciertas circunstancias significó contravenir la opinión de los expertos y mantener algunas variantes, tras especificar dicha salvedad en un apartado de observaciones de la ficha terminológica.

En general, estas dificultades fueron percibidas por los estudiantes como inherentes a la realización de cualquier trabajo terminológico. Por la misma razón, solucionar los distintos problemas constituye uno de los componentes de la competencia con respecto a la variación terminológica y, por lo tanto, forma parte de los logros de aprendizaje del proyecto.

\subsubsection{Evaluación acerca de la utilidad del trabajo en la formación académica: encuesta}

La opinión de los estudiantes respecto a la utilidad de la tarea en su formación se recabó mediante un cuestionario en línea anónimo ${ }^{6}$, administrado a todos los estudiantes del grupo (nro. $=49)$. En primer lugar, se solicitó que evaluaran la utilidad del trabajo en relación con los objetivos de aprendizaje del proyecto y de la asignatura de Terminología 3. En la Tabla II se puede apreciar que los estudiantes consideran que el trabajo les sirvió para comprender la importancia de la variación terminológica en la traducción e interpretación (100 \%) y tomar conciencia de la variación terminológica dialectal en el lenguaje especializado (98\%).

6 El cuestionario se puede consultar en el siguiente link: https://docs.google.com/ forms/d/e/1FAIpQLScKkjBs_CXFXhD3N8wxp1dbgUMW5dgDCFiN0ggJcAcQ20rSOQ/ viewform? $\mathrm{vc}=0 \& \mathrm{c}=0 \& \mathrm{w}=1$ [Consulta: $21 / 12 / 2019]$. 
Tabla II. Valoración de los estudiantes acerca de la utilidad del trabajo en su formación.

\begin{tabular}{|l|c|c|c|c|c|}
\hline Criterios & $\begin{array}{l}\text { Muy en } \\
\text { desacuerdo }\end{array}$ & En desac. & $\begin{array}{l}\text { Ni ac. } \\
\text { ni desac. }\end{array}$ & $\begin{array}{l}\text { De } \\
\text { acuerdo }\end{array}$ & $\begin{array}{l}\text { Muy de } \\
\text { acuerdo }\end{array}$ \\
\hline $\begin{array}{l}\text { Entender la impor- } \\
\text { tancia de la variación } \\
\text { terminológica para la } \\
\text { T\&I. }\end{array}$ & $0 \%$ & $0 \%$ & $0 \%$ & $29 \%$ & $71 \%$ \\
\hline $\begin{array}{l}\text { Aprender sobre termi- } \\
\text { nología médica. }\end{array}$ & $0 \%$ & $2 \%$ & $12 \%$ & $20 \%$ & $65 \%$ \\
\hline $\begin{array}{l}\text { Tomar conciencia de } \\
\text { la variación termino- } \\
\text { lógica dialectal en el } \\
\text { lenguaje especializado. }\end{array}$ & $0 \%$ & $2 \%$ & $0 \%$ & $18 \%$ & $80 \%$ \\
\hline $\begin{array}{l}\text { Aprender la metodo- } \\
\text { logía del trabajo ter- } \\
\text { minológico puntual }\end{array}$ & $0 \%$ & $2 \%$ & $10 \%$ & $31 \%$ & $57 \%$ \\
\hline
\end{tabular}

Por un lado, entre los comentarios de los estudiantes, algunos señalaron que "antes no había pensado siquiera que existía este fenómeno" y que, gracias al proyecto, "valoramos más que antes el fenómeno de la variación dialectal". Por otro lado, algunos estudiantes declararon que, si bien esta variación está presente en todos los campos, "pudimos notar la importancia de esta en un área tan especializada como lo es la medicina". Finalmente, el trabajo también les sirvió para aprender respecto a la funcionalidad de la variación, ya que "al encontrar nuevas variantes se puede aprender mucho cómo denominar algo a través de nuevas maneras" y la relevancia de una gestión adecuada de este fenómeno en el proceso traductor, puesto que "hace comprender la importancia de documentarse bien al momento de realizar un trabajo". Asimismo, un $88 \%$ de los estudiantes estimó que el proyecto les sirvió para instruirse en la metodología del trabajo terminológico puntual. De este modo, un estudiante manifestó que "fue muy interesante observar que detrás de un glosario terminológico existe un gran trabajo de investigación previo" y valoraron que el aprendizaje se llevara a cabo en un contexto real, ya que "es necesario aplicar esa metodología a un trabajo real para poder aprenderlo bien". 


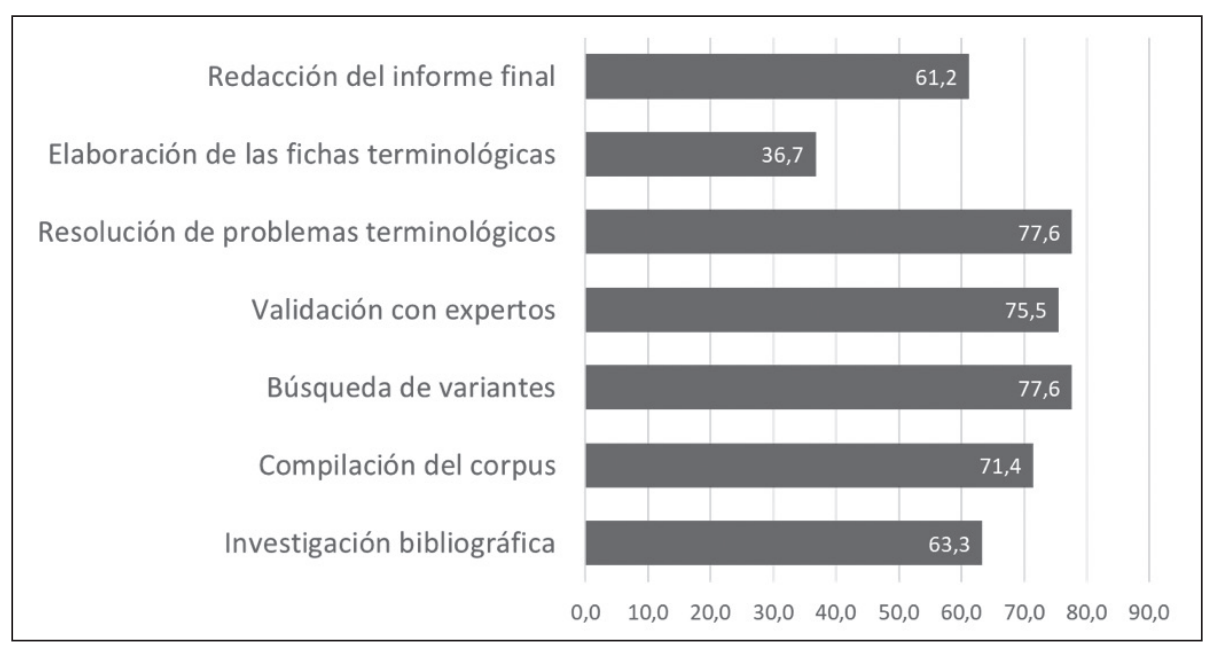

Figura 3. Fases del trabajo terminológico de mayor utilidad según los estudiantes.

También se les preguntó acerca de qué etapas del proyecto consideraban más útiles para su formación. Como se aprecia en la figura 3, fueron las fases de búsqueda de variantes $(77,6 \%)$ y de resolución de problemas terminológicos $(77,6$ $\%)$ las que encontraron más útiles, seguidas de la tarea de validación de las variantes con especialistas de medicina $(75,5 \%)$ y de compilación del corpus con textos y recursos terminológicos de medicina específicos del español de Chile $(71,4 \%)$. Las etapas de búsqueda bibliográfica para elaborar el marco teórico y la redacción del informe final fueron consideradas de menor utilidad (en torno al $62 \%$ de los estudiantes las seleccionaron). Por último, la fase de diseño y llenado de las fichas terminológicas fue la peor valorada $(36,7 \%)$, por el carácter mecánico y minucioso de la tarea.

Finalmente, los estudiantes valoraron muy positivamente el carácter situado de la tarea, concretamente que el resultado de su trabajo esté disponible en VariMed y que se enmarque en un proyecto de colaboración científica. Como muestra la figura 4, un $100 \%$ de los estudiantes evaluaron positivamente la colaboración científica con una universidad extranjera, puesto que "siempre es un placer saber que se contribuye de alguna manera a la comunidad". También valoraron que su trabajo sirva para la investigación en traducción e interpretación, dado que "la investigación es un área que debería fomentarse, por ende, me gusta que el trabajo que he realizado sirva para este fin". Asimismo, un 93,9\% consideró valiosa esta 
experiencia académica para su currículum y también estuvo de acuerdo en que participar en una investigación real en el marco de una colaboración científica otorgaba sentido a la tarea, ya que "saber que formará parte de un proyecto oficial incita a realizar un trabajo bien hecho" y "hace sentir que tanto trabajo será valorado".

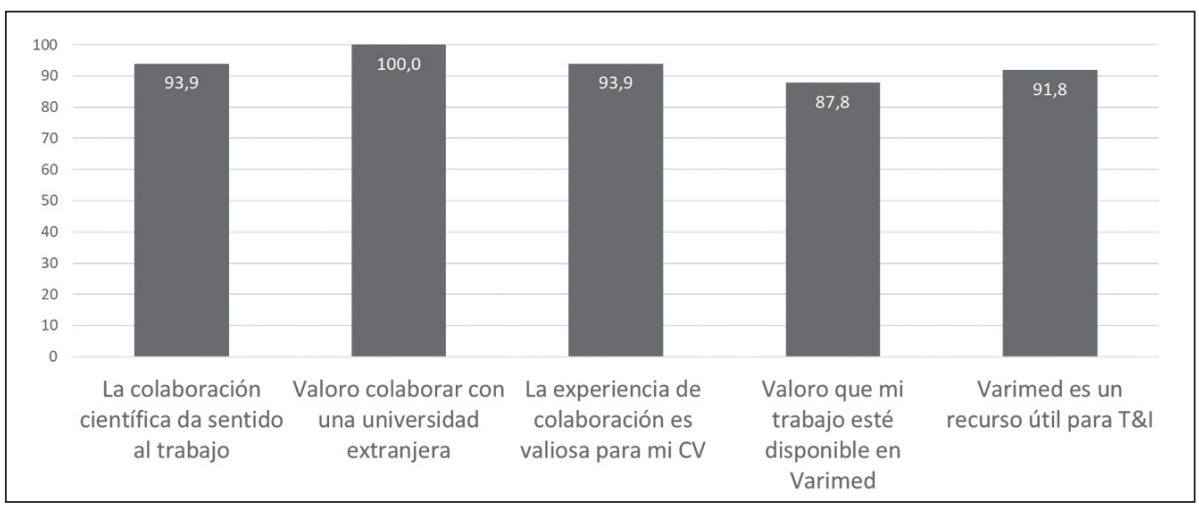

Figura 4. Valoración de los estudiantes respecto al carácter situado del trabajo.

Por último, un $87,8 \%$ evaluó positivamente que las fichas de las variantes documentadas en Chile estén disponibles para su consulta en VariMed y un 91,8 $\%$ estimó que era un recurso útil para traductores e intérpretes. Un/a estudiante declaró que "VariMed puede convertirse en un recurso primario para los traductores del área médica" y otro valoró que se incluyan variantes no solo de España, ya que "como traductores e intérpretes de Chile, tendríamos que hacer una documentación similar a la que se realizó en este trabajo para comprobar el uso de la(s) variante(s) en el país".

En síntesis, los resultados del cuestionario muestran que la evaluación del trabajo fue altamente positiva por parte de los estudiantes, además de que el proyecto sirvió efectivamente para desarrollar conocimiento acerca del fenómeno de la variación terminológica y habilidades para su adecuada gestión a través de glosarios, una de las competencias terminológicas imprescindibles para traductores e intérpretes. La mayoría de las dificultades mencionadas se concentran en la fase de documentación, de identificación de variantes y de validación con expertos, lo cual constituye un insumo crucial con el fin de revisar la propuesta didáctica y trabajar estos aspectos con mayor tiempo y énfasis en las clases de terminología. 


\section{CONCLUSIONES}

La competencia terminológica es parte esencial de las habilidades del traductor y los enfoques situados ofrecen un ambiente adecuado para el desarrollo de dicha competencia, puesto que ponen en contacto al estudiante con diferentes entornos socioculturales, comunicativos y cognitivos donde se aplican las nociones terminológicas. Uno de los aspectos de la terminología que más relevancia posee desde una perspectiva situada es la variación, pues enfrenta al estudiante a un problema frecuente y a menudo ignorado en la teoría y enseñanza de la terminología, cuya gestión requiere un análisis profundo de los parámetros sociogeográficos y situacionales en los que se desenvuelve la comunicación especializada en dos sistemas lingüísticos distintos y el conocimiento de las distintas fases del trabajo terminológico para su correcta documentación y representación en los recursos terminológicos.

En este trabajo se han descrito los resultados de una experiencia colaborativa entre la Universidad de Granada y la Pontificia Universidad Católica de Valparaíso, para el desarrollo de la subcompetencia sobre variación terminológica en estudiantes de traducción e interpretación de la universidad chilena. La colaboración entre universidades en dos continentes ha supuesto una toma de conciencia acerca de los aspectos comunes que marcan el trabajo en la traducción, especialmente aquellos que están relacionados con la variación desde una perspectiva geolectal. Las competencias y habilidades documentales y terminológicas de los estudiantes se potencian con una propuesta de implementación real, tal como constituye la llevada a cabo en este proyecto con respecto al recurso VariMed.

\section{REFERENCIAS}

Bowker, Lynne. (2015). Terminology and translation. En H. J. Kockaert y F. Steurs (Eds.), Handbook of terminology: volume 1. Amsterdam, Philadelphia: John Benjamins, 304-323.

Brown, John; Collins, Allan y Duguid, Paul. (1989). Situated Cognition and the Culture of Learning. Educational Researcher, 18 (1), 32-42.

Buendía, Miriam y Ureña, José Manuel. (2010). ¿Cómo diseñar un corpus de calidad? Parámetros de evaluación. Sendebar. 21, pp. 165-180.

Cabré, Teresa. (1999). La terminología: representación y comunicación: elementos para una teoría de base comunicativa y otros artículos. Barcelona, España: IULAUPF.

Cabré, Teresa y Tebé, Carles. (2005). El trabajo terminológico multilingüe de enriquecimiento lingüístico: una propuesta metodológica. Revista española de lingüistica aplicada. 1, pp. 19-41. 
Calsamiglia, Helena y Van Dijk, Teun. (2004). Popularization discourse and knowledge about the genome. Discourse and society. 15 (4), pp. 369-389.

Collet, Tanja. (2004). Esquisse d'une nouvelle microstructure de dictionnaire spécialisé reflétant la variation en discours du terme syntagmatique. Meta: Journal des traducteurs. 49 (2), pp. 247-263.

Daille, Béatrice; Habert, Benoît; Jacquemin, Christian y Royauté, Jean. (1996). Empirical observation of term variations and principles for their description. Terminology. 3(2), pp. 197-258.

Diéguez, María; Lazo, Rosa y Tebé, Carles. (2016). La traducción en Chile: resultados del estudio de mercado 2015. Santiago, Chile: Facultad de Letras, Pontificia Universidad Católica de Chile.

Fernández, Sabela. (2016). The cognitive and rhetorical role of term variation and its contribution to knowledge construction in research articles. Terminology. 22 (1), pp. 52-79.

Fernández, Sabela. (2018). The cognitive and communicative functions of term variation in research articles: a comparative study in Psychology and Geology. Applied Linguistics. 40(4), pp. 624-645.

Fernández, Sabela y Becerra, Nelson. (2015). La variación terminológica en la comprensión y producción de textos académicos: Propuesta de representación en un diccionario especializado de aprendizaje de Psicología. Revista Ibérica. 20, pp. 183-208.

Fernández, Sabela; Freixa, Judit y Cabré, María. (2011). A proposed method for analysing the dinamics of cognition through term variation. Terminology. 17 (1), pp. 49-74.

Freixa, Judit. (2006). Causes of denominative variation in terminology: a typology proposal. Terminology. 12 (1), pp. 51-77.

Gerding, Constanza; Fuentes, Mary y Kotz, Gabriela. (2012). Anglicismos y aculturación en la sociedad chilena. Onomázein: Revista de lingüistica, filología y traducción. 25 (1), pp. 139-162.

Gómez, Adelina y Vargas, Chelo. (2003). Metodología para alimentar una base de datos terminológica desde las necesidades del traductor. Actas del I Congreso Internacional de la Asociación Ibérica de Estudios de Traducción e Interpretación, Granada: AIETI, pp. 629-648.

Gouadec, Daniel. (2007). Translation as a Profession. Amsterdam/Philadelphia: John Benjamins.

Hendricks, Cher. (2001). Teaching Causal Reasoning Through Cognitive Apprenticeship: What Are Results From Situated Learning? The Journal of Educational Research. 94 (5), pp. 302-311.

Hurtado, Amparo. (2007). Traducción y Traductología: Introducción a la traductología. (2da edic.). Madrid: Cátedra.

Kelly, Dorothy. (2005). A Handbook for Translator Trainers. A Guide to Reflective 
Practice. Manchester: St. Jerome Publishing.

Kerremans, Koen. (2010). A comparative study of terminological variation in specialized translation. En Heine y Engberg (Eds.), Reconceptualizing LSP. Online proceedings of the XVII European LSP Symposium. Aarhus: Aarhus School of Business, pp. 1-14.

Kiraly, Donald. (2000). A Social Constructivist Approach to Translator Education: Empowerment from Theory to Practice. Manchester, Northampton: St. Jerome Publishing.

Montero, Silvia y Faber, Pamela. (2009). Terminological Competence in Translation. Terminology. 15 (1), pp. 88-104.

Montero, Silvia; Faber, Pamela y Buendía, Miriam. (2011). Terminología para traductores e intérpretes. Granada, España: Ediciones Tragacanto.

Navarro, Fernando A. (2001). El inglés, idioma internacional de la medicina. Panace@. 3 (2), pp. 35-51.

Olvera, María Dolores; Robinson, Bryan; Castro, Rosa; Quero Gervilla, Enrique; Muñoz Martín, Ricardo; Muñoz, Eva; Murillo, Miguel; Senso, José; Vargas, Benjamín y Díez Lerma, José. (2007). A professional Approach to Translator Training (PATT). Meta: Journal des traducteurs. 52 (3), pp. 517-528.

Prieto, Juan y Fuentes, Adrián. (2016). A collaborative multimodal working environment for the development of instrumental and professional competences of student translators: an innovative teaching experience. The Interpreter and Translator Trainer. 10 (1), pp. 76-91.

Risku, Hanna. (2002). Situatedness in Translation Studies. Cognitive Systems Research, 3 (3), 523-533.

Risku, Hanna. (2016). Situated learning in translation research training: academic research as a reflection of practice. The Interpreter and Translator Trainer. 10 (1), pp. 12-28.

Robinson, Bryan J.; López, Clara y Tercedor, Maribel. (2008). Neither Born nor Made, but Socially-Constructed: Promoting Interactive Learning in an Online Environment. TTR: Traduction, Terminologie, Rédaction, 21 (2), 95-129.

Sánchez, Pilar; Aguilar, Anna; Mesa, Bartalomé y Solé, Marta. (2009). Applying Terminology Knowledge to Translation: Problem-based Learning for a Degree in Translation and Interpreting. Terminology. 15 (1), pp. 105-118.

Suárez, María. (2004). Análisis contrastivo de la variación denominativa en textos especializados: del texto original al texto meta. Tesis de Doctorado en Lingüística Aplicada. Barcelona, España: Institut Universitari de Lingüística Aplicada, Universitat Pompeu Fabra.

Tercedor, Maribel. (2011). The cognitive dynamics of terminological variation. Terminology. 17 (2), pp. 181-197.

Tercedor, Maribel. (2017). Bases cognitivas de la variación terminológica en medicina. En M. A. Candel Mora y Ch. Vargas-Sierra, Temas actuales de termino- 
logía y estudios sobre el léxico. Granada: Comares, pp. 27-48.

Tercedor, Maribel; Faber, Pamela y López, Clara. (2012). Working with Words: Research Approaches in Translation-oriented Lexicographic Practice. TTR: Traduction, Terminologie, Rédaction. 25 (1), pp. 181-214.

Ulrych, Marguerita. (2003). Diversity, uniformity and creativity in translation. En Petrilli (Ed.), Translation. Translation. Amsterdam/New York: Brill Rodopi, pp. 133-151.

Umaña, Olga y Suárez, María. (2011). Descripción y explicación del diseño de instrumentos que miden la competencia traductora y terminología en traductores profesionales. Revista EAN. 70, pp. 22-41.

Ünal, Cezmi y Zeynep, Hatice. (2010). Students' Perceptions of a Situated Learning Environment. Procedia: Social and Behavioral Sciences. 2, pp. 2171-2175.

Vienne, Jean. (1994). Toward a pedagogy of 'translation in situation'. Perspectives. 2 (1), 51-59.

Vygotsky, Lev. (1978). Mind in society: The development of higher psychological processes. Cambridge, MA: Harvard University Press. 